\title{
Dispersion forces in inhomogeneous planarly layered media: A one-dimensional model for effective polarizabilities
}

\author{
Johannes Fiedler, ${ }^{1,2}$ Fabian Spallek, ${ }^{1}$ Priyadarshini Thiyam, ${ }^{3}$ Clas Persson, ${ }^{2}$ Mathias Boström, ${ }^{2,4}$ \\ Michael Walter, ${ }^{1,5,6}$ and Stefan Yoshi Buhmann ${ }^{1,7}$ \\ ${ }^{1}$ Physikalisches Institut, Albert-Ludwigs-Universität Freiburg, Hermann-Herder-Strasse 3, D-79104 Freiburg im Breisgau, Germany \\ ${ }^{2}$ Centre for Materials Science and Nanotechnology, Department of Physics, University of Oslo, P.O. Box 1048 Blindern, \\ NO-0316 Oslo, Norway \\ ${ }^{3}$ Division of Theoretical Chemistry, Lund University, P.O. Box, S-22100 Lund, Sweden \\ ${ }^{4}$ Department of Energy and Process Engineering, Norwegian University of Science and Technology, NO-7491 Trondheim, Norway \\ ${ }^{5}$ FIT Freiburg Centre for Interactive Materials and Bioinspired Technologies, Georges-Köhler-Allee 105, 79110 Freiburg, Germany \\ ${ }^{6}$ Fraunhofer IWM, Wöhlerstrasse 11, D-79108 Freiburg i. Br., Germany \\ ${ }^{7}$ Freiburg Institute for Advanced Studies, Albert-Ludwigs-Universität Freiburg, Albertstr. 19, D-79104 Freiburg, Germany
}

(Received 23 August 2018; published 25 June 2019)

\begin{abstract}
Dispersion forces such as van der Waals forces between two microscopic particles, the Casimir-Polder forces between a particle and a macroscopic object, or the Casimir force between two dielectric objects are well studied in vacuum. However, in realistic situations the interacting objects are often embedded in an environmental medium. Such a solvent influences the induced dipole interaction. With the framework of macroscopic quantum electrodynamics, these interactions are mediated via an exchange of virtual photons. Via this method the impact of a homogeneous solvent medium can be expressed as local-field corrections leading to excess polarizabilities which have previously been derived for hard boundary conditions. In order to develop a more realistic description, we investigate a one-dimensional analog system illustrating the influence of a continuous dielectric profile.
\end{abstract}

DOI: 10.1103/PhysRevA.99.062512

\section{INTRODUCTION}

Dispersion forces are among the weakest forces in nature and are caused by the ground-state fluctuations of the vacuum electromagnetic field [1]. In contrast to the theory developed by Casimir [2] which deals with the Casimir force between two dielectric plates in vacuum, his work originally was motivated by a colloidal system - the stabilization of hydrophobic suspensions of particles in dilute electrolytes [3]. In this description, the field fluctuations induce dipole moments inside the considered objects which can interact with other materials. Alternative accounts derive dispersion forces from position dependent ground-state energies of the coupled field-matter system $[2,4]$. The dispersion forces resulting from this process are classified by three different types of interacting partners: the Casimir force which describes the interaction of two neutral macroscopic dielectric objects, the van der Waals force acting between two polarizable particles and the CasimirPolder force connecting both by describing the interaction of a polarizable particle with a macroscopic dielectric body. During recent years these forces have been well studied in several experiments [5-8] and in theory [2,9-11]. However, most of these investigations were restricted to laboratory conditions by using vacuum chambers which remove any environment. In colloidal physics, experiments and theoretical models for medium assisted local-field corrections [12,13] were based on the simplest model by Onsager [14,15] including a cavity surrounding the particles. Note that local-field effects have been extensively studied in the context of spontaneous emission $[12,13]$.

In natural situations, where these effects play an important role, such as the Gecko feet [16] or colloids [17], this assumption is not valid any more. In principle, one can assume that an environmental medium results in a decrease of the interaction due to the screening caused by the medium of permittivity $\varepsilon$. This is expressed via a factor $\varepsilon^{-1}$ leading to the expected decrease of the potential. From fundamental quantum mechanics it is known that a particle embedded in a medium has no immediate contact with the medium particles. Caused by Pauli repulsion, a small vacuum layer is formed surrounding the particle [18]. With respect to dispersion forces, the impacts of an environment can be separated into two classes: the screening of particle's internal electrostatic wave functions, which leads to a change of the optical properties [19], and geometrical change of electromagnetic wave scattering. The latter impact will be considered in this manuscript. This has given rise to the development of a cavity model [15] describing the boundaries of both medium and particle as hard, discontinuous changes in the permittivity. Different models for the local-field corrections are expressed in terms of an effective polarizability; a spherical two- or three-layer system is considered for Onsager's real cavity model and the finitesize model, respectively. In the two-layer case the particle is treated to be pointlike in the center and the optical response is modified by the transmission of light through the boundary following Mie reflection [Fig. 1(a)]. For larger objects, such as 


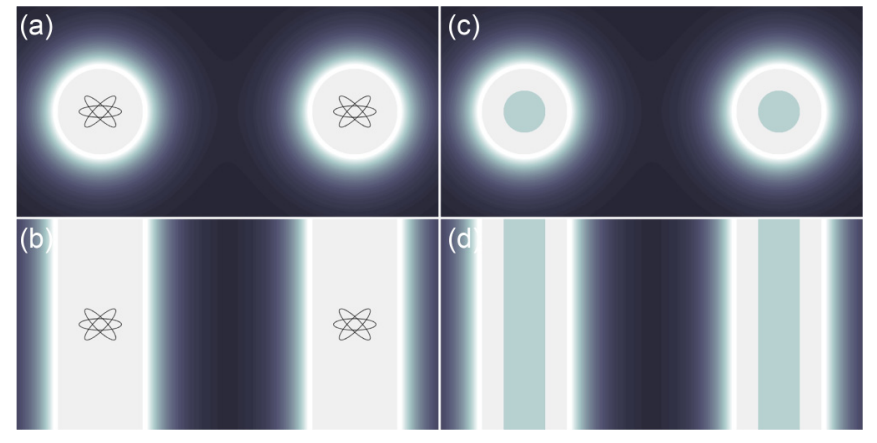

FIG. 1. Sketch of the considered setups for the spherical problem and the one-dimensional analogon: (a) two particles embedded in a medium creating an Onsager's real cavity with inhomogeneous dielectric profile; (b) one-dimensional analogon with planarly inhomogeneous profile; (c) two spherical nanoparticles embedded in a medium with an inhomogeneous cavity; (d) the corresponding onedimensional problem with two dielectric plates of finite thickness embedded in a medium with inhomogeneous profile.

clusters or molecules, the particle's extension has to be taken into account which will be modeled by replacing the point particle with an additional layer [Fig. 1(c)]. In this case, the excess polarizability is determined by the reflection of light at the outer boundary. However, exact microscopic investigations have shown that these boundaries have to be described by continuous profiles [20], which have been numerically investigated [21].

In this manuscript, we analyze the influence of continuous boundary profiles on dispersion forces. For simplicity we consider planarly layered systems leading to an effective one-dimensional model. Figure 1 illustrates the corresponding models which represent two particles interacting via van der Waals forces. In Fig. 1(a) the particles are embedded in a medium and create an inhomogeneous boundary profile. The corresponding one-dimensional situation is depicted in (b), where we consider two planar cavities with continuous boundaries and centered particles interacting with each other. Figure 1(c) illustrates the finite-size cavity model with an inhomogeneous boundary. The corresponding situation consists of two plates of finite thickness embedded in a planar cavity; see Fig. 1(d).

We hence consider two scenarios: two particles or two plates in a five-layer system, representing the van der Waals and the Casimir force, respectively. In these two cases, we cover all important wave propagations at an inhomogeneous profile - the transmission through and the reflection at a continuous profile.

\section{CASIMIR FORCE AND VAN DER WAALS POTENTIAL}

Figures 2(a) and 2(b) sketch a cross section along the direction perpendicular to the layers for the van der Waals and Casimir cases, respectively. Two objects are embedded in a medium of permittivity $\varepsilon_{2}$ and create a cavity with a vacuum permittivity $\varepsilon_{1}=1$. We focus on the impact of the continuous boundary conditions on the dispersion forces. To this end, we repeat the important steps of the theory via scattering Green's functions, derive the Green's functions for the layered case,

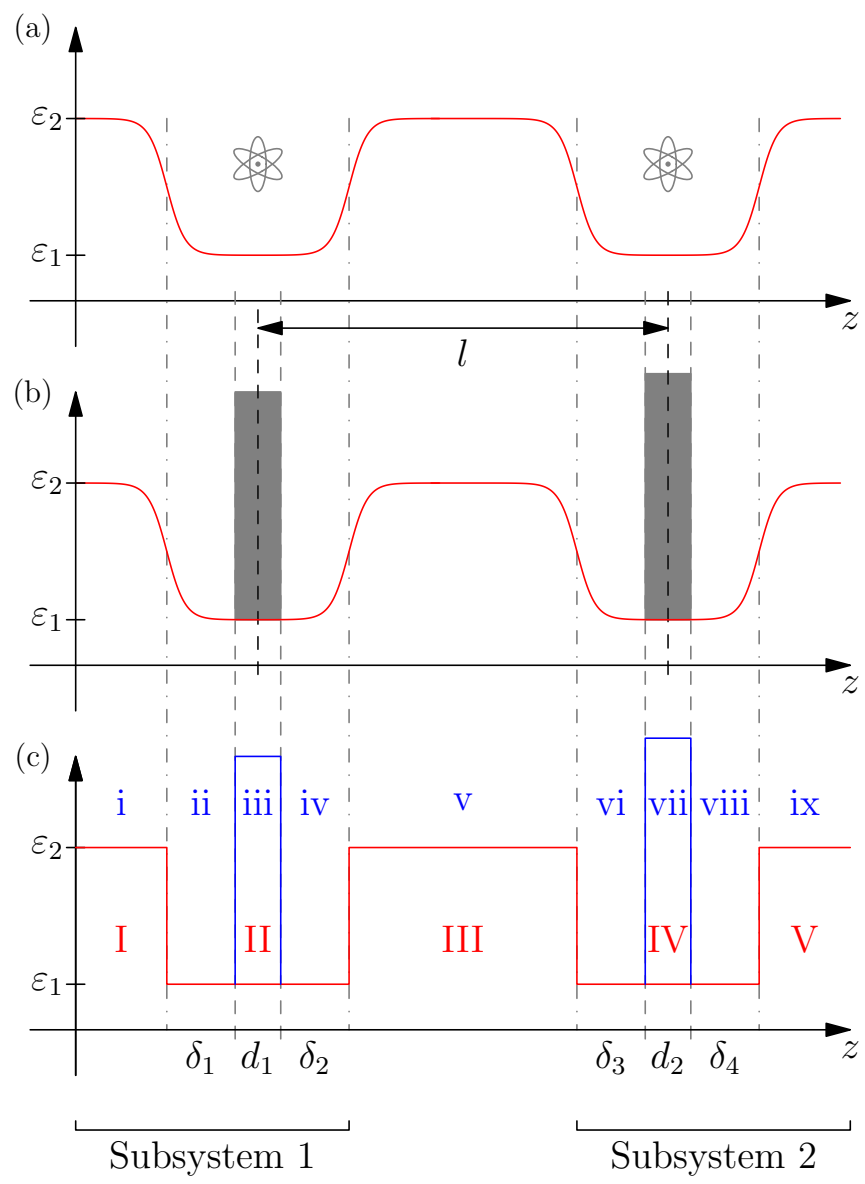

FIG. 2. Sketch of the corresponding one-dimensional dielectric profiles for (a) the van der Waals force, where two particles are separated by a distance $l$ and embedded in a continuous dielectric cavity (red curve) and (b) the Casimir force, where the two particles have been exchanged by slabs of thicknesses $d_{2}$. The third picture (c) illustrates the corresponding cases with hard boundaries, which is for the van der Waals force a five-layer system labeled with capital roman numbers (I-V) and for the Casimir force a nine-layer system (small roman numbers i-xi).

and calculate the impact of the cavity in terms of a local field correction in analogy to the known excess polarizabilities. Finally, we model the reflection at a continuous dielectric profile and illustrate the effect by applying the method to example profiles.

The Casimir force can be obtained from the electromagnetic stress tensor [11] as a surface integral over the considered body. The Abraham-Minkowski stress tensor can be obtained in terms of dyadic Green's function [17]

$$
\begin{aligned}
\mathbf{T}(\mathbf{r})= & -\frac{\hbar}{\pi} \int_{0}^{\infty} d \xi\left\{\frac{\xi^{2}}{c^{2}} \varepsilon(i \xi) \mathbf{G}(\mathbf{r}, \mathbf{r}, i \xi)+\frac{\tilde{\mathbf{G}}(\mathbf{r}, \mathbf{r}, i \xi)}{\mu(i \xi)}\right. \\
& \left.-\frac{1}{2} \operatorname{tr}\left[\frac{\xi^{2}}{c^{2}} \varepsilon(i \xi) \mathbf{G}(\mathbf{r}, \mathbf{r}, i \xi)+\frac{1}{\mu(i \xi)} \tilde{\mathbf{G}}(\mathbf{r}, \mathbf{r}, i \xi)\right]\right\},
\end{aligned}
$$


where $\tilde{\mathbf{G}}(\mathbf{r}, \mathbf{r}, \omega)$ denotes the contributions from the magnetic field

$$
\tilde{\mathbf{G}}(\mathbf{r}, \mathbf{r}, \omega)=\nabla \times \mathbf{G}\left(\mathbf{r}, \mathbf{r}^{\prime}, \omega\right) \times\left.\nabla^{\prime}\right|_{\mathbf{r}^{\prime} \rightarrow \mathbf{r}},
$$

where the final derivative acts to the left. For infinite plates located in the $x-y$ plane it is sufficient to introduce the force density per unit area

$$
\mathbf{f}=\frac{d \mathbf{F}}{d A}=\mathbf{e}_{z} \cdot\left[\mathbf{T}(z=b)-\mathbf{T}\left(z=b^{\prime}\right)\right]
$$

where $b$ and $b^{\prime}=b-d_{1}$ denote the positions of the right and left boundary of the considered plate, respectively. The dyadic Green function $\mathbf{G}\left(\mathbf{r}, \mathbf{r}^{\prime}, \omega\right)$ is the fundamental solution of the vector Helmholtz equation for purely dielectric systems $(\mu=$ 1) $[10]$

$$
\left[\nabla \times \nabla \times-\frac{\omega^{2}}{c^{2}} \varepsilon(\mathbf{r}, \omega)\right] \mathbf{G}\left(\mathbf{r}, \mathbf{r}^{\prime}, \omega\right)=\delta\left(\mathbf{r}-\mathbf{r}^{\prime}\right) .
$$

A similar method can be applied to calculate the van der Waals interaction. We consider two polarizable particles at positions $\mathbf{r}_{1}$ and $\mathbf{r}_{2}$ with polarizabilities $\alpha_{1}$ and $\alpha_{2}$. By applying fourth-order perturbation theory to the interaction Hamiltonian $H=-\hat{\mathbf{d}}_{1} \cdot \hat{\mathbf{E}}\left(\mathbf{r}_{1}\right)-\hat{\mathbf{d}}_{2} \cdot \hat{\mathbf{E}}\left(\mathbf{r}_{2}\right)$ the van der Waals potential can be found as [11]

$$
\begin{aligned}
U_{\mathrm{vdW}}\left(\mathbf{r}_{1}, \mathbf{r}_{2}\right)= & -\frac{\hbar \mu_{0}^{2}}{2 \pi} \int_{0}^{\infty} d \xi \xi^{4} \alpha_{1}(i \xi) \alpha_{2}(i \xi) \\
& \times \operatorname{tr}\left[\mathbf{G}\left(\mathbf{r}_{1}, \mathbf{r}_{2}, i \xi\right) \cdot \mathbf{G}\left(\mathbf{r}_{2}, \mathbf{r}_{1}, i \xi\right)\right]
\end{aligned}
$$

By comparing Eqs. (1) and (5) one observes that Casimir force requires the coincidence limit $\left(\mathbf{r}^{\prime} \rightarrow \mathbf{r}\right)$, whereas the van der Waals potential depends on the Green's tensor at two positions. Thus the Casimir force is governed by the reflections of virtual photons and the van der Waals force by their transmissions.

To address the influence of an inhomogeneous dielectric profile on the van der Waals force, we separate the profile into nine sections as illustrated in Fig. 2(a): five regions with spatially constant dielectric function and four with continuous profiles. The impact of the regions with continuous dielectric function will be reduced to generalized reflection and transmission coefficients entering the five layer problem, layers I-V in Fig. 2(c).

We start our investigations by neglecting the inhomogeneous profile regions. As mentioned before the important quantities are the transmission and reflections at these interfaces, which leads us to write the influence of the cavities in terms of reflection coefficients and to perform the transition to the cavities with continuous profiles via exchanging the hardboundary reflection coefficients with the ones determined for a continuous dielectric profile. The situation for the Casimir force is nearly the same. However, in this case we have to consider two additional layers [layers iii and vii in Fig. 2(c)] corresponding to the plates representing the interacting objects and we have to calculate the reflection coefficients at both sides of one of these plates, e.g., the left one illustrated in Figs. 2(b) and 2(c).

\section{GREEN'S FUNCTIONS FOR PLANAR MULTILAYERED SYSTEMS AND LOCAL FIELD CORRECTIONS FOR PLANAR CAVITIES}

In order to construct the scattering processes in the considered scenario, we start with a discontinuous multilayered system with general reflection and transmission coefficients and perform the transition to the continuous profile by substituting the reflection coefficients, which will be derived in Sec. IV. We assume the width of the environment around the particles to be small compared to the separation of the particles, $d_{1}, d_{2} \ll l$, and that the latter is small enough such that the interaction is nonretarded. This allows us to approximate the Green's function for the five-layer system in terms of a local-field corrected bulk Green's function.

The Green's function of a system involving inhomogeneous media can be described by the bulk Green's function modified with the local field correction factors arising from the reflection and transmission of the electromagnetic field through the various layers of inhomogeneities of the system [11]. That is, for the five-layer system considered here, the full Green's tensor can be written as, similar to the local-field correction for cavities [22],

$$
\begin{aligned}
\mathbf{G}\left(\mathbf{r}, \mathbf{r}^{\prime}, \omega\right)= & \left.\int \frac{d^{2} \mathbf{k}^{\|}}{(2 \pi)^{2}} e^{-i \mathbf{k}^{\|} \cdot\left(\varrho-\varrho^{\prime}\right)} \tilde{t}\left(\mathbf{k}^{\|}\right)\right|_{\mathbf{r}} \\
& \times\left.\cdot \mathbf{G}^{(1)}\left(z, z^{\prime}, \mathbf{k}^{\|}, \omega\right) \cdot \tilde{t}\left(\mathbf{k}^{\|}\right)\right|_{\mathbf{r}^{\prime}},
\end{aligned}
$$

where $\left.\tilde{t}\right|_{\mathbf{r}}$ and $\left.\tilde{t}\right|_{\mathbf{r}^{\prime}}$ represent the generalized transmission coefficients close to the final and source point, respectively, while $\mathbf{G}^{(1)}\left(\mathbf{r}, \mathbf{r}^{\prime}, \omega\right)$ denotes the scattering Green's tensor for a bulk system. Equation (6) can be understood as the Weyl expansion of the planar Green's function, modified transmission coefficients. We used cylindrical coordinates $\mathbf{r}=(\varrho, z)$. The transmission and reflection coefficients marked with a tilde denote the complete ones including multiple reflections, whereas the ones without a tilde are the ordinary Fresnel coefficients at a single interface.

In order to estimate the complete van der Waals interaction, we write down the scattering Green's function for a single interface and obtain the multiple reflection coefficients for the multiple reflections. The scattering Green's tensor for a source situated in layer 1 and field in layer $2\left(z^{\prime}<z\right)$ in the nonretarded limit is given by [11]

$$
\begin{aligned}
\mathbf{G}\left(\mathbf{r}, \mathbf{r}^{\prime}, i \xi\right)= & \frac{c^{2}}{8 \pi \xi^{2} \sqrt{\varepsilon_{1} \varepsilon_{2}}} \\
& \times \int_{0}^{\infty} d \kappa e^{-\kappa\left(z-z^{\prime}\right)} t_{12} \kappa^{2} \operatorname{diag}(1,1,-2),
\end{aligned}
$$

where we have used $\kappa=\kappa_{1}^{\perp}=\kappa_{2}^{\perp}=k^{\|}$. Further, in the nonretarded limit, the transmission coefficient simplifies to the Fresnel coefficient $[11,23,24]$

$$
t_{12}=\frac{2 \varepsilon_{1}}{\varepsilon_{1}+\varepsilon_{2}},
$$

and consequently it becomes independent from the wave vector $k$. By further considering a homogeneous medium, one can set $\varepsilon_{1}=\varepsilon_{2}$, meaning the transmission coefficient simplifies to 


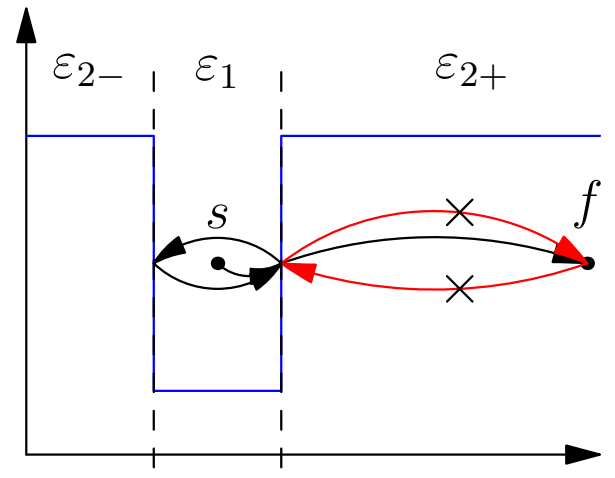

FIG. 3. Sketch of the three layer system with a centered medium $\varepsilon_{1}$ surrounded by a right $\varepsilon_{2+}$ and left medium $\varepsilon_{2-}$ illustrating the considered optical paths from the source point $s$ in the centered layer to the final point $f$ far in the right layer. One possible path is marked in black including the multiple scattering inside the cavity. The multiple scattering between the final point and the outer interface between the right and centered medium will not be considered due to the large separation.

1 and the integral in Eq. (7) can be performed [11]:

$$
\mathbf{G}\left(\mathbf{r}, \mathbf{r}^{\prime}, i \xi\right)=\frac{c^{2}}{4 \pi \xi^{2} \varepsilon} \frac{1}{\left(z-z^{\prime}\right)^{3}} \operatorname{diag}(1,1,-2) .
$$

We can compare this result with the nonretarded bulk Green's tensor [11]

$$
\mathbf{G}\left(\mathbf{r}, \mathbf{r}^{\prime}, i \xi\right)=\frac{c^{2}}{4 \pi \xi^{2} \varepsilon s^{3}}\left[\mathbf{I}-3 \mathbf{e}_{s} \mathbf{e}_{s}\right],
$$

with the three-dimensional unit matrix I, the relative coordinate $\mathbf{s}=\mathbf{r}-\mathbf{r}^{\prime}$, its absolute value $s=|\mathbf{s}|$, and its unit vector $\mathbf{e}_{s}=\mathbf{s} / s$. By again setting $x=x^{\prime}=y=y^{\prime}=0$ in Eq. (10), we indeed recover Eq. (9). Thus the results are consistent.

Coming back to the original situation, which is the multilayer case, we consider the three-layer system depicted in Fig. 3. The aforementioned two-layer system (of infinite thickness with single interface in between) describes our fundamental way of treating a single interface. By assuming a large central layer we can neglect the multiple scattering inside this layer due to the strong reduction upon propagation, $\propto e^{-\kappa_{2} l}$. Thus we can construct the complete transmission via the product of the transmission coefficient entering the middle layer $t_{12}$ and exiting it $t_{23}$, leading us to identify the generalized transmission coefficients from Eq. (6):

$$
\left.\tilde{t}\right|_{\mathbf{r}}=t_{12},\left.\quad \tilde{t}\right|_{\mathbf{r}^{\prime}}=t_{23} .
$$

Assuming that both particles are the same, the shape of the cavities will also match each other and the transmission out of the middle layer can be transformed to

$$
t_{23}=1+r_{23}=1-r_{32}=2-t_{32},
$$

where we used the relation $t=1+r$ between reflection and transmission coefficients for a layered system, which is only valid for a sharp interface. Thus, for a symmetric profile, we further simplify the generalized transmission coefficients to

$$
\left.\tilde{t}\right|_{\mathbf{r}}=t_{12},\left.\quad \tilde{t}\right|_{\mathbf{r}^{\prime}}=2-t_{12}
$$

In order to adapt the transmission through a two-layer system to the sought-after five-layer scenario, the transmission coefficient has to be modified with respect to multiple reflections at the additional interfaces. Again, we restrict our consideration to multiple reflections only inside the cavity with width $d$. For a single cavity, it is sufficient to consider a three-layer system, where a centered medium with dielectric function $\varepsilon_{1}$ and width $d$ is in contact with two infinitely extended layers with dielectric functions $\varepsilon_{2 \pm}$ (where " + " denotes the right medium and "-" the left medium; see Fig. 3). We consider a particle to be centered in the middle layer and estimate the transmission of an electromagnetic wave created at the particle into the right medium $\varepsilon_{2+}$. All optical paths starting inside the cavity and terminating in this medium can be written as

$$
\begin{aligned}
\tilde{t}_{12+}= & t_{12+}+r_{12+} p r_{12-} p t_{12+} \\
& +r_{12+} p r_{12-} p r_{12+} p r_{12-} p t_{12+}+\cdots \\
& +r_{12-} p t_{12+}+r_{12-} p r_{12+} p r_{12-} p t_{12+}+\cdots,
\end{aligned}
$$

with the propagation along the cavity $p=e^{-\kappa d}$. The first term denotes the part which is directly transmitted, the second line denotes all odd parts, which are initially sent towards the right interface, and the third line denotes the even parts, which start with a reflection at the left interface before all are transmitted into the third layer. This equation can be recast as a geometric series

$$
\begin{aligned}
\tilde{t}_{12+} & =\left[1+r_{12-} e^{-\kappa d}\right]\left[\sum_{n=0}^{\infty}\left(r_{12-} r_{12+} e^{-2 \kappa d}\right)^{n}\right] t_{12+} \\
& =\frac{1+r_{12-} e^{-\kappa d}}{1-r_{12-} r_{12+} e^{-2 \kappa d}} t_{12+},
\end{aligned}
$$

where again the first term collects even and odd paths, the second term is the sum over all multiple reflections, and finally the transmission into the right layer. By considering the left and right medium to be equal $\varepsilon_{2-}=\varepsilon_{2+} \equiv \varepsilon$ the local-field corrections simplify to

$$
\tilde{t}=\frac{1+r}{1-r e^{-\kappa d}}
$$

with the Fresnel reflection coefficient $r=(\varepsilon-1) /(\varepsilon+1)$. In the limit of vanishing cavity size $(d \rightarrow 0)$ the local-field correction can be further simplified to

$$
\tilde{t}=\frac{1+r}{1-r}=\varepsilon
$$

Note that this solution results in a discontinuity when comparing with the homogeneous medium case. Summarizing, the local-field correction, Eq. (6), reads

$$
\begin{aligned}
\mathbf{G}\left(\mathbf{r}, \mathbf{r}^{\prime}, \omega\right)= & \int_{0}^{\infty} d \kappa e^{-i \kappa\left(z-z^{\prime}\right)} \frac{1+r}{1-r e^{-\kappa d}} \\
& \times \mathbf{G}^{(1)}\left(z, z^{\prime}, \kappa, \omega\right)\left(2-\frac{1+r}{1-r e^{-\kappa d}}\right),
\end{aligned}
$$


and can be further simplified to

$$
\mathbf{G}\left(\mathbf{r}, \mathbf{r}^{\prime}, \omega\right)=\varepsilon(2-\varepsilon) \int_{0}^{\infty} d \kappa e^{-i \kappa\left(z-z^{\prime}\right)} \mathbf{G}^{(1)}\left(z, z^{\prime}, \kappa, \omega\right)
$$

by assuming a vanishing cavity. In order to describe the corresponding situation for the Casimir force, we again start with the scattering Green's function for a two layered system. When considering an inhomogeneous profile, the generalized transmission coefficient reads

$$
\left.\tilde{t}\right|_{\mathbf{r}}=\frac{\tau}{1-\rho e^{-\kappa d}}
$$

where $\rho$ denotes the reflection coefficient at and $\tau$ the transmission coefficient through the inhomogeneous region, because the relation $t=1+r$ is not valid anymore. Analogously, one finds

$$
\left.\tilde{t}\right|_{\mathbf{r}^{\prime}}=\frac{\tilde{\tau}}{1+\rho e^{-\kappa d}}
$$

with the transition coefficient for the opposite direction $\tilde{\tau}$. To this end, the local-field corrected Green's function for inhomogeneous boundaries reads as

$$
\begin{aligned}
\mathbf{G}\left(\mathbf{r}, \mathbf{r}^{\prime}, \omega\right)= & \int_{0}^{\infty} d \kappa e^{-i \kappa\left(z-z^{\prime}\right)} \\
& \times \frac{\tau(\kappa) \tilde{\tau}(\kappa)}{1-\rho^{2}(\kappa) e^{-2 \kappa d}} \mathbf{G}^{(1)}\left(z, z^{\prime}, \kappa, \omega\right) .
\end{aligned}
$$

In contrast to the van der Waals case, the source and observation points have to be located in the same layer in this case. Hence we begin our calculation with a similar expression for the Green's function and need to replace the transmission coefficients by the corresponding expression for reflections, leading to [11]

$$
\begin{aligned}
\mathbf{G}\left(\mathbf{r}, \mathbf{r}^{\prime}, \omega\right)= & \frac{i}{8 \pi^{2}} \int_{0}^{\infty} \frac{d^{2} k^{\|}}{k^{\perp}} \\
& \times e^{i \mathbf{k}^{\|} \cdot\left(\mathbf{r}-\mathbf{r}^{\prime}\right)-i\left(k_{2}^{\perp} z-k_{1}^{\perp} z^{\prime}\right)} r_{12} \mathbf{e}_{1}^{+} \mathbf{e}_{1}^{-},
\end{aligned}
$$

where in the nonretarded limit the reflection coefficient for an $s$-polarized wave vanishes directly for a dielectric medium.

In this case, we have to consider a nine-layer system depicted in Fig. 2(c) and estimate the Casimir pressure acting on the left plate. In Fig. 2(c) the two plates of thickness $d_{1}$ and $d_{2}$ are illustrated as layers iii and vii. Each of them is located in a cavity marked by layers ii and iv with thickness $\delta_{1}$ and $\delta_{2}$ for the left plate and layers vi and viii with thickness $\delta_{3}$ and $\delta_{4}$. Layers $i$ and ix have an infinite thickness. Both subsystems are separated by the middle layer $\mathrm{v}$ of thickness $l$. Before we continue with the estimation of the local-field correction for this case, we recall the motivation for this analysis which is the estimation of the impact of a continuous dielectric profile for the one-dimensional analog of the real cavity models. This means that the two subsystems consisting of the layers $i$ to iv and of the layers vi to iv are fixed, as depicted in Fig. 2(c). Thus we can treat these two subsystems as two effective layers and can map the situation onto the well-known case of a planar cavity resulting in the simple expression for the Casimir force
[11]

$$
\mathbf{f}=\frac{\hbar}{2 \pi^{2}} \int_{0}^{\infty} d \xi \int_{0}^{\infty} d k^{\|} \kappa^{\perp} k^{\|} \frac{r^{+} r^{-} e^{-2 \kappa^{\perp} l}}{1-r^{+} r^{-} e^{-2 \kappa^{\perp} l}} \mathbf{e}_{z},
$$

where $r^{ \pm}$denotes the outside reflection coefficients for $p$ polarized waves at the right, which is subsystem 2 (consisting of the layers vi-ix), and left interface, which is subsystem 1 (layers i-iv), according to the three-layer system discussed for the van der Waals force. Thus the initial and final points for this consideration are located in the centered layer v. Further, we used the fact that the reflection coefficients for $s$-polarized waves vanish.

Let us consider the local-field correction for the left subsystem 1 (layers $\mathrm{i}$ to iv). In terms of the nine-layer system the effective reflection coefficient at the interface between the fifth and the fourth layer has to be determined. It will be constructed iteratively starting with effective reflection between the third and second interface [24]

$$
\tilde{r}_{32}=r_{32}+\frac{t_{32} r_{21} t_{23} e^{-\kappa_{\mathrm{ii}} \delta_{1}}}{1-r_{32} r_{23} e^{-2 \kappa_{\mathrm{ii}} \delta_{1}}},
$$

where $\kappa_{\mathrm{ii}}$ denote the imaginary part of the wave vector in the second layer. Starting from this effective reflection coefficient, one can continue with the next interface

$$
\tilde{r}_{43}=\frac{r_{43}+\tilde{r}_{32} e^{-2 \kappa_{\mathrm{iii}} d_{1}}}{1-r_{43} \tilde{r}_{32} e^{-2 \kappa_{\mathrm{iii}} d_{1}}}
$$

and finally

$$
\tilde{r}_{54}=\frac{r_{54}+\tilde{r}_{43} e^{-2 \kappa_{\mathrm{iv}} \delta_{2}}}{1-r_{54} \tilde{r}_{43} e^{-2 \kappa_{\mathrm{iv}} \delta_{2}}},
$$

with the wave vectors in the third and fourth layer denoted as $\kappa_{\mathrm{iii}}$ and $\kappa_{\mathrm{iv}}$, respectively. Assuming that $\varepsilon_{\mathrm{ii}}=\varepsilon_{\mathrm{iv}}=1$ and $\varepsilon_{\mathrm{i}}=\varepsilon_{\mathrm{v}}=\varepsilon$ leads to equal wave vectors in the corresponding layers and equal distances for the cavity layers $\left(\kappa_{\mathrm{ii}}=\kappa_{\mathrm{iv}}=\kappa\right.$ and $\delta_{1}=\delta_{2}=\delta$ ) and thus only two elementary reflection coefficients are important: the one between the cavity and the slab $r_{23}=r_{43}=r_{1}$ and between the cavity and the medium $r_{21}=r_{45}=r$. Then, the generalized reflection coefficient simplifies to

$$
\begin{aligned}
\tilde{r}= & -\frac{1+r_{1}^{2} e^{-2 \kappa \delta}}{r_{1}^{4} e^{-4 \kappa \delta}+r r_{1}^{3} e^{-3 \kappa \delta}-r r_{1} e^{-3 \kappa \delta}+2 r_{1}^{2} e^{-2 \kappa \delta}+1} \\
& \times\left[r^{2} r_{1}\left(r_{1}^{2}-1\right) e^{-2 \kappa_{\mathrm{III}} d_{1}-\kappa \delta}+\left(r r_{1}^{4}+r_{1}\right) e^{-2 \kappa_{\mathrm{III}} d_{1}-2 \kappa \delta}\right. \\
& +\left(r r_{1}^{2}-r\right) e^{-2 \kappa_{\mathrm{III}} d_{1}-3 \kappa \delta}+r_{1}^{3} e^{-2 \kappa_{\mathrm{III}} d_{1}-4 \kappa \delta} \\
& \left.+\left(r r_{1}^{2}-r_{1}\right) e^{-2 \kappa \delta}+r_{1}^{2} r e^{-2 \kappa_{\mathrm{III}} d_{1}}-r_{1}^{3} e^{-4 \kappa \delta}+r\right] \\
& \times\left[r_{1}^{4} e^{-2 \kappa_{\mathrm{III}} d_{1}-2 \kappa \delta}+r_{1}^{3} r e^{-2 \kappa_{\mathrm{III}} d_{1}-\kappa \delta}+r_{1}^{2} e^{-2 \kappa \delta}\right. \\
& \left.-r_{1} r e^{-2 \kappa_{\mathrm{III}} d_{1}-\kappa \delta}+r_{1}^{2} e^{-2 \kappa_{\mathrm{III}} d_{1}}+1\right]^{-1} .
\end{aligned}
$$

The generalized reflection coefficient at the right interface can be determined analogously leading to the same result by exchanging the reflection coefficient at the slab to the other materials $r_{1}=\left(\varepsilon_{1}-1\right) /\left(\varepsilon_{1}+1\right) \mapsto r_{2}=\left(\varepsilon_{2}-1\right) /\left(\varepsilon_{2}+1\right)$. In case of an inhomogenous boundary at the interfaces between layers i and ii and layers iv and v, the transmission coefficients in Eq. (25) do not simplify and the generalized 
reflection coefficient reads as

$$
\begin{aligned}
\tilde{r}= & r_{1}\left[-\left(r_{1}^{4}+r_{1}\right) e^{-2 \kappa_{\mathrm{iii}} d_{1}-2 \kappa \delta}+\rho r_{1} \tau \tilde{\tau} e^{-2 \kappa_{\mathrm{iii}} d_{1} \kappa \delta}\right. \\
& +\rho \tau \tilde{\tau} e^{-2 \kappa_{\mathrm{iii}} d_{1}-3 \kappa \delta}-r_{1}^{3} e^{-2 \kappa_{\mathrm{iii}}-4 \kappa \delta} \\
& \left.-r_{1}^{2}\left(e^{-2 \kappa \delta}+e^{-2 \kappa_{\mathrm{iii}} d_{1}}\right)-1\right]\left[r_{1}^{3}\left(r_{1}-1\right) e^{-2 \kappa_{\mathrm{iii}} d_{1}-2 \kappa \delta}\right. \\
& +\rho r_{1}^{2} \tau \tilde{\tau} e^{-2 \kappa_{\mathrm{iii}} d_{1}-\kappa \delta}-r_{1}^{5} e^{-2 \kappa_{\mathrm{iii}} d_{1}-4 \kappa \delta} \\
& \left.-\rho r_{1} \tau \tilde{\tau} e^{-2 \kappa_{\mathrm{iii}} d_{1}-\kappa \delta}+r_{1}^{2}\left(e^{-2 \kappa \delta}+e^{-2 \kappa_{\mathrm{iii}} d_{1}}\right)+1\right]^{-1},
\end{aligned}
$$

where the Greek letters denote the reflection coefficient $\rho$ and transmission coefficients $\tau, \tilde{\tau}$ at the inhomogeneous boundary.

Now, we restrict ourselves to the case that both slabs consist of equal materials, leading to the same reflection coefficient.

\section{REFLECTION AT AN INHOMOGENEOUS BOUNDARY}

With respect to an arbitrary one-dimensional spatial susceptibility profile, $\varepsilon(z, \omega)$, the resulting reflection coefficient can be obtained by solving the Riccati differential equation [24]

$$
\begin{aligned}
R^{\prime}(z)= & -2 \kappa_{0} \sqrt{\varepsilon(z, \omega)+\left(\frac{k_{\|}}{\kappa_{0}}\right)^{2}} R(z) \\
& -\frac{1}{4} \frac{\varepsilon^{\prime}(z, \omega)}{\varepsilon(z, \omega)} \frac{2 k_{\|}^{2}+\kappa_{0}^{2} \varepsilon(z, \omega)}{k_{\|}^{2}+\kappa_{0}^{2} \varepsilon(z, \omega)}\left[1-R^{2}(z)\right],
\end{aligned}
$$

for $p$-polarized waves, with $\kappa_{0}=\omega / c$. We omit the discussion of $s$-polarized waves, because they vanish in the nonretarded limit. This equation strictly follows from the system of equations of an $n$-layered system (25)-(27) by increasing the number of layers $n$ and then decreasing their widths to zero $d \rightarrow 0$. We restrict ourselves to the case of infinite support for the dielectric profile $\varepsilon(z)$ with $z \in \mathbb{R}$ and consider the field's source and final points to be located at $+\infty$. Thus, for a given profile $\varepsilon(z)$, the solution of the Riccati differential equation gives the reflection coefficient $R(z)$ at the position $z$ for a wave coming from the right side. For more details on this formalism see the thorough account found in the textbook [24]. This equation has a unique solution with the initial condition $R(z \rightarrow-\infty)=0$, which means initially no reflection and gives rise to the condition for the considered profile that the gradient should vanish at the initial point $\lim _{z \rightarrow 0} \varepsilon^{\prime}(z)=0$. Analogously, we find the left sided reflection coefficients by using the relation

$$
R^{-}(z)=-R^{+}(z)
$$

Figure 4 illustrates the different profiles of inhomogeneity, where we want to describe the reflection process. Due to the spatial dependence of the profile the point of reflection changes by the distance $a$ which we assume to be equal for all types of profiles and define its value by the crossing point of a linear profile reaching the final value as depicted in Fig. 4.

Again, we apply the nonretarded limit to estimate the reflection coefficients. Then the Riccati differential equation

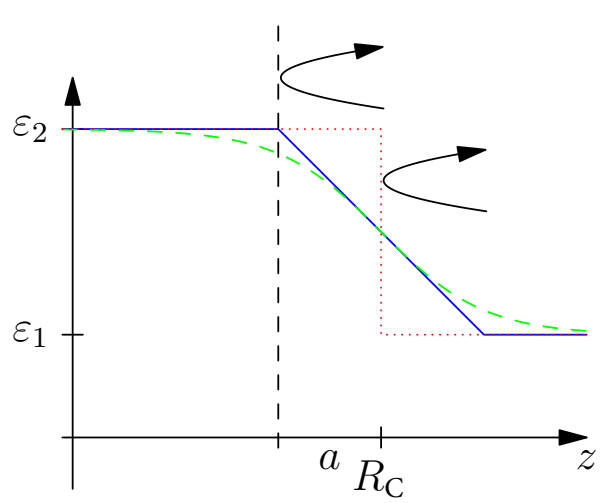

FIG. 4. Sketch of the different spatial dependent dielectric functions for the hard boundary (red dotted curve), the linear profile (blue curve), and the nonlinear profile (green dashed curve). The black arrows illustrate the considered reflections which take place for the hard boundary at the cavity radius $R_{\mathrm{C}}$ and for the functional profiles at their ends which are shifted by a distance $a$ compared to the hard-boundary case.

simplifies to

$$
R^{\prime}(z)=-2 k_{\|} R(z)-\frac{1}{2} \frac{\varepsilon^{\prime}(z, \omega)}{\varepsilon(z, \omega)}\left[1-R^{2}(z)\right] .
$$

This equation can be solved analytically for vanishing wave vector $\left(k_{\|}=0\right)$ and results in

$$
R(z)=\tanh \left\{-\frac{1}{2}[\ln \varepsilon(z, \omega)-\ln \varepsilon(-a, \omega)]\right\},
$$

which can be simplified further to

$$
R\left(z_{\text {end }}\right)=\frac{\varepsilon_{2}(\omega)-\varepsilon_{1}(\omega)}{\varepsilon_{2}(\omega)+\varepsilon_{1}(\omega)}
$$

by assuming that the profile connects both dielectric functions as depicted in Fig. 4. This result denotes the ordinary Fresnel reflection coefficient in agreement to the considered case of nonpropagating waves. Due to this fact, we can determine the impact of the linear term describing the screening of light by the propagation through the finite profile of the length $2 a$.

Corresponding to the profile plotted in Fig. 4, we choose two different spatial profiles for the dielectric function.

(i) A linear function

$$
\begin{aligned}
\varepsilon(z, \omega)= & \varepsilon_{1}(\omega)+\left[\varepsilon_{2}(\omega)-\varepsilon_{1}(\omega)\right] \\
& \times \begin{cases}1 & \text { for } z<-a, \\
\frac{a-z}{2 a} & \text { for }|z| \leqslant a, \\
0 & \text { otherwise }\end{cases}
\end{aligned}
$$

and (ii) a Thomas-Fermi distributed profile

$$
\varepsilon(z, \omega)=\varepsilon_{1}(\omega)+\left[\varepsilon_{2}(\omega)-\varepsilon_{1}(\omega)\right]\left(1+e^{\frac{2 z}{a}}\right)^{-1},
$$

approximating typical profiles; see Ref. [20]. Both profiles have the same slope at the origin $(z=0)$. The width of the inhomogeneous profiles are $2 a$. Thus the profiles end at the point $a$. The boundary conditions are for case (i) $R(-a)=0$ and for case (ii) $R(z \rightarrow-\infty)=0$.

Numerical solutions of the Riccati differential equation (32) are depicted in Fig. 5 where we evaluate the reflection 


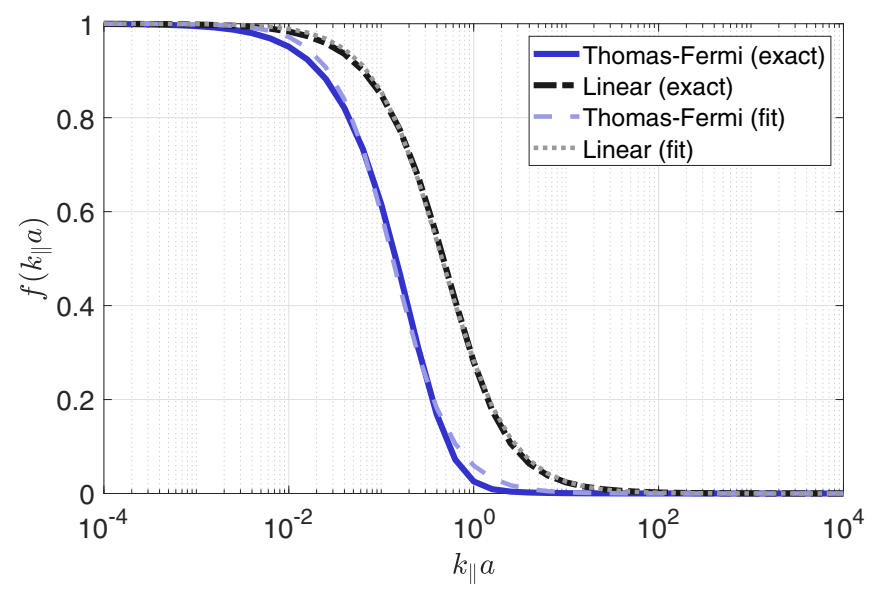

FIG. 5. Geometric dependence of the reflection coefficients for both profiles-Thomas-Fermi and linear-with the exact solution of the Riccati (solid blue and dashed black lines) equation and the corresponding approximations (dashed light blue and dotted gray lines).

coefficient at the end of the inhomogeneous profile. For the linear profile, this is the distance $a$, and for the ThomasFermi distribution we increased the final point to $3 a$ reaching the final value with a deviation of less than $1 \%$. It can be observed that the $k_{\|}$dependence is related to the thickness of the inhomogeneous region $2 a$. Several checks with different parameters showed that the resulting curves only depend on the product of the wave vector and the corresponding length scale of the inhomogeneity. The depicted results can be understood by introducing the wavelength $\lambda=2 \pi / k_{\|}$leading to the ratio of the wavelength and the inhomogeneity's thickness as the relevant quantity. It can be seen that if this ratio is small $a / \lambda<1$ the specific profile does not matter to the result and the solution behaves like a hard boundary. In the other case where the ratio is large $a / \lambda>1$ the reflection decreases to zero. The interesting region is denoted by the case when the wavelength is comparable to the thickness $\lambda \approx a$. From the numerical simulations one can conclude that the final reflection coefficient separates into a product of two terms: one describes the dielectric properties and the other the geometric properties

$$
\rho\left[k_{\|}, \varepsilon_{1}(\omega), \varepsilon_{2}(\omega), a\right]=\frac{\varepsilon_{2}-\varepsilon_{1}}{\varepsilon_{2}+\varepsilon_{1}} \cdot f\left(k_{\|} a\right),
$$

satisfying the $k_{\|} \rightarrow 0$ limit, Eq. (34). For both investigated cases the expressions of the result are the same with different parameters. In analogy to the solution of the Riccati equation, Eq. (33), and in agreement with the numerical results depicted in Fig. 5, we approximate the $k_{\|}$dependence of the reflection coefficient by

$$
f\left(k_{\|} a\right) \approx \frac{1}{2}\left[1-\tanh \left(\frac{\ln \left(k_{\|} a\right)-\lambda_{1}}{\lambda_{2}}\right)\right] .
$$

The resulting parameters are given in Table I, which perfectly match the curves with a coefficient of determination of $\approx$ $100 \%$ for the linear profile and of $99.96 \%$ for the ThomasFermi distribution. The results are in agreement with other
TABLE I. Fitting parameter for both profiles (linear and ThomasFermi distributed) based on the approximation equation (38).

\begin{tabular}{lcc}
\hline \hline Profile & $\lambda_{1}$ & $\lambda_{2}$ \\
\hline Linear & -0.555 & 2.028 \\
Thomas-Fermi & -2.067 & 1.452 \\
\hline \hline
\end{tabular}

studies [25-28] solving Maxwell's equations for the linear profile.

\section{RESULTS AND DISCUSSION}

Applying the local-field correction (6) to the van der Waals potential (5) one finds

$$
\begin{aligned}
U_{\mathrm{vdW}}\left(z, z^{\prime}\right)= & -\frac{3 \hbar}{64 \pi^{3} \varepsilon_{0}^{2}} \int_{0}^{\infty} d \xi \frac{\alpha_{1}(i \xi) \alpha_{2}(i \xi)}{\varepsilon_{1}(i \xi) \varepsilon_{2}(i \xi)} \\
& \times \int_{0}^{\infty} d \kappa d \kappa^{\prime} \kappa^{2} e^{-\kappa\left(z-z^{\prime}\right)} \frac{\tilde{\tau}(\kappa) \tau(\kappa)}{1-\rho^{2}(\kappa) e^{-2 \kappa d}} \\
& \times \kappa^{\prime 2} e^{-\kappa^{\prime}\left(z-z^{\prime}\right)} \frac{\tilde{\tau}\left(\kappa^{\prime}\right) \tau\left(\kappa^{\prime}\right)}{1-\rho^{2}\left(\kappa^{\prime}\right) e^{-2 \kappa^{\prime} d}}
\end{aligned}
$$

with the transmission coefficients at the inhomogeneous boundary $\tau$ and $\tilde{\tau}$ and the reflection coefficients $\rho$ have to be evaluated for the inhomogeneous profile which is modeled by Eqs. (37) and (38) for the reflection. We assume that the transmission through the inhomogeneous region can be modeled with the Fresnel transmission through a step profile due to the short width of the layer (hence $t=1+r$ ), as we explicitly showed for the $s$-wave transmission; see the Appendix.

In order to illustrate the theory we consider helium atoms solved in water. Helium's polarizability was taken from Ref. [29] and the dielectric function for water from Ref. [30]. The resulting dielectric profile is depicted in Fig. 6, which is a position-dependent dielectric function. A density can be

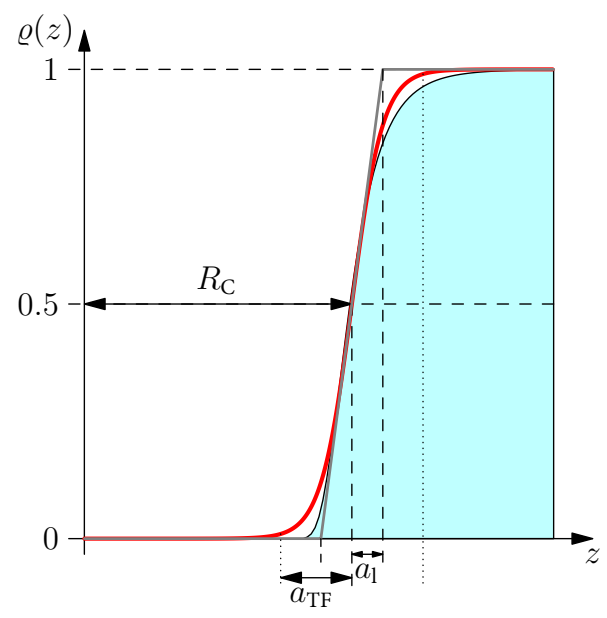

FIG. 6. Spatial density profile of water surrounding a helium atom with the cavity radius $R_{\mathrm{C}}$. Simulated profile enclosed the blue area which is approximated by a Thomas-Fermi distribution (red line) with the corresponding thickness of the inhomogeneous region $2 a_{\mathrm{TF}}$ and by a linear profile (gray line) with the thickness $2 a_{\mathrm{l}}$. 


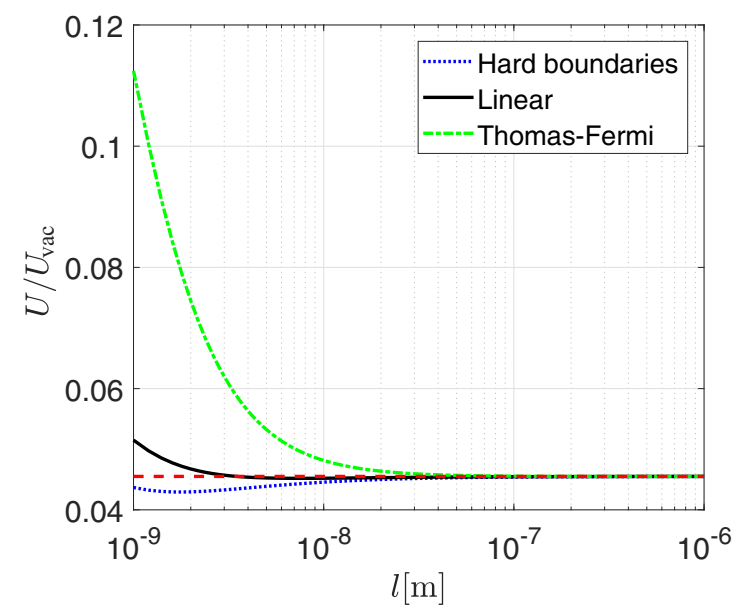

FIG. 7. Relative van der Waals potentials for the cavity model with hard boundaries (blue dotted line), linear boundaries (black solid line), and Thomas-Fermi distributed boundary profiles (green dashed-dotted line) with respect to the vacuum potential. The general reduction of the potential caused by the screening in water (factor $\approx 20$ ) can be observed (red dashed line).

extracted from the simulation via

$$
\varepsilon(z, \omega)=1+\varrho(z)[\varepsilon(\omega)-1],
$$

in analogy to the examples given in Eqs. (35) and (36) with vacuum on one side $\left[\varepsilon_{1}(\omega)=1\right]$. Note that the density is not a particle density. The simulated profile is fitted to a ThomasFermi distribution

$$
\varrho(z)=\left(1+e^{-\alpha\left(z-R_{\mathrm{C}}\right)}\right)^{-1},
$$

with the cavity radius $R_{\mathrm{C}}=1.71 \AA$ and the profile's slope $\alpha=$ $10.1 \AA^{-1}$, via a least-squares algorithm. A comparison of this function with the profiles used to solve the Riccati differential equation (36) relates the fitting parameter $\alpha$ to the generalized profile width $a_{1}=2 / \alpha$ directly denoting the thickness for the linear profile. Using the same value to determine the thickness of the Thomas-Fermi distributed profile would result in an underestimation of the thickness, because one would only take into account $73 \%$ of the profile's amplitude. This can be improved by increasing the layer size. To do so, we define the thickness in this case by a threshold of $99 \%\left[\varrho\left(a_{\mathrm{TF}}\right)=0.99\right]$ leading to $a_{\mathrm{TF}}=-\ln (0.0101) / \alpha$. The resulting parameters are $0.198 \AA$ for the linear profile and $0.455 \AA$ for the ThomasFermi profile. Relating these parameters to fitted profiles depicted in Fig. 5 one can upper bound for the relevant wave vectors of $k_{\max } \approx 5 \times 10^{10} \mathrm{~m}^{-1}$ for the linear profile and $k_{\max } \approx 2 \times 10^{10} \mathrm{~m}^{-1}$ for the Thomas-Fermi profile.

The van der Waals potentials for helium atoms in water are depicted in Fig. 7. A planarly layered cavity profile surrounding two helium atoms are considered, which has an equivalent dimension as the spherical cavity. This means that the atoms are $1.71 \AA$ behind the water-vacuum interfaces. In the figure the dependent quantity is the thickness of the intermediate layer $l$. This leads to a changed power law for shorter separation in the order of the particle interface distance. In order to compare the results are added for the vacuum potential and the hard-boundary potential to discuss

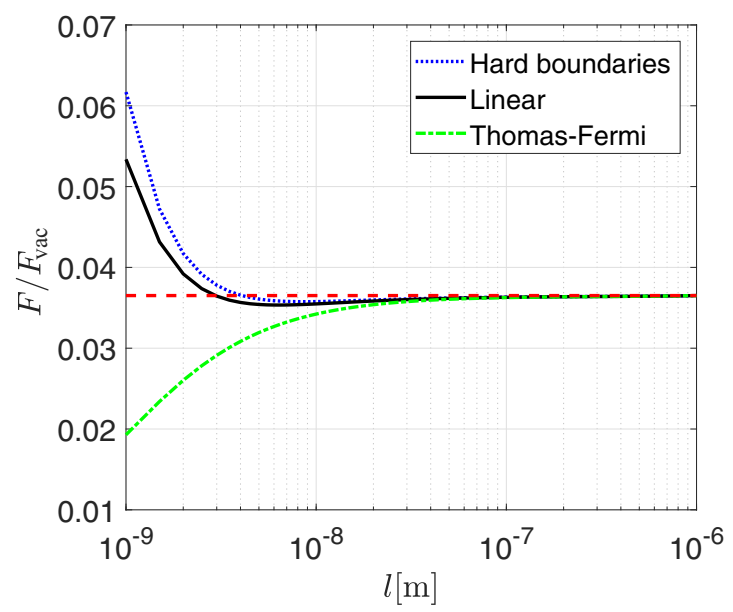

FIG. 8. Relative impact of the cavity boundaries on the Casimir force for a helium plate embedded in water relative to the force in vacuum. Without any cavity corrections (dashed red line), with hard boundaries (doted blue line), with the linear profile (solid black line), and with the Thomas-Fermi distributed profile (dashed-dotted green line).

the impact. It can be observed that the presence of the medium reduces the potential, which is obvious due to screening inside a medium. However, a softer boundary increases the potential at short distances. At larger distances the impact of the boundary's shape vanishes, which is caused by long wavelengths dominating the scattering in this region. It can be seen further that a smoother profile leads to a stronger increase of the interaction potential.

For the Casimir force, we apply the method to a similar example, where we replace the helium atoms by two helium plates of finite thickness $d_{1}=d_{2}=d=1.14 \AA$ [31-33]. The dielectric function of these plates can be estimated via the Clausius-Mossotti relation [15,34]

$$
\varepsilon=\frac{1+2 \alpha /\left(4 \pi \varepsilon_{0} d^{3}\right)}{1-\alpha /\left(4 \pi \varepsilon_{0} d^{3}\right)},
$$

with the polarizability of a helium atom $\alpha$, entering the reflection coefficient

$$
r_{1}=\frac{\varepsilon-1}{\varepsilon+1}
$$

By approximating the reflection coefficients for the cavities via Eq. (37) and applying the result to the local-field corrected Casimir force, Eq. (24) together with Eq. (29), the Casimir force acting on two helium plates in water can be obtained. The results are depicted in Fig. 8. The general reduction of the Casimir force caused by the presence of the environmental medium is illustrated by the dashed red line. Further, the reduction of the interaction at short distances due to the finite size of the cavity can be seen as well. The influence of the profile shape is located in the region for short distances when the profile lengths $a$ and the separation of the plate distances $l$ are comparably large. Here, one observes a further reduction which is stronger for the Thomas-Fermi distributed profile than for the linear profile. The strong reduction which is observed in these cases is caused by the assumed size of the atomic layer. Many known values will not fulfill the Clausius- 
Mossotti relation. Thus we chose a value at the upper bound of the error deviation for the van der Waals radius satisfying this relation. However, this implies a stronger dielectric function for solid helium than it has to be, which further decreases the interaction caused by its presence in the local-field reflection coefficient, Eq. (29), $\tilde{r}=\tilde{r}\left(\kappa_{\text {iii }}\right)$.

\section{CONCLUSIONS}

The aim of this article was the illustration of the impact of a cavity with a continuous boundary profile on dispersion interactions in media. To this end, we investigated an equivalent situation with a one-dimensional cavity and derived the local-field correction induced by the cavity simplifying the theory to be applicable for known two- and three-layer cases. Further, we approximated the Fresnel reflection coefficient at a continuous profile with a fitted function which is valid for the cases of a linear profile or a Thomas-Fermi distributed profile. To illustrate the impact on the relevant dispersion forces we applied the method to the case of two helium atoms in water (van der Waals potential) and for two helium plates embedded in water (Casimir force). We found that the influence is relevant at small distances. On larger scales the impact of the continuous profile vanishes and the hard-boundary case is a suitable description. To account for more realistic three-dimensional systems, an adaptation of this theory to three-dimensional spherical objects is required, which will be the subject of further investigations.

\section{ACKNOWLEDGMENTS}

We would like to thank an anonymous referee for discussions. We gratefully acknowledge support by the German Research Foundation (Grant No. BU1803/3-1, S.Y.B. and J.F.), the Research Innovation Fund by the University of Freiburg (S.Y.B., J.F., and M.W.), and the Freiburg Institute for Advanced Studies (S.Y.B.). We acknowledge financial support from the Research Council of Norway (Project No. 250346) and from COST Action MP1406 'MultiscaleSolar' (STSM Reference No. 39969).

\section{APPENDIX: TRANSMISSION OF $s$ WAVES THROUGH AN INHOMOGENEOUS BOUNDARY}

A wave propagating along a continuous planar layered system which is described by a dielectric function $\varepsilon(z)$ can be described by the scalar Helmholtz equation

$$
\left[\frac{d^{2}}{d z^{2}}+k_{0}^{2} \varepsilon(z)-k_{\|}^{2}\right] \varphi(z)=0,
$$

with $k_{0}=\omega / c$. For the linear profile the equation transforms into a harmonic oscillator outside the inhomogeneous region

$$
\left[\frac{d^{2}}{d z^{2}}+k_{0}^{2} \varepsilon_{1}-k_{\|}^{2}\right] \varphi^{<-a}(z)=0 \quad \begin{cases}\text { for } & z<-a, \\ \text { for } & z>a\end{cases}
$$

and

$$
\left[\frac{d^{2}}{d z^{2}}+A+B z\right] \varphi(z)=0
$$

with $A=\frac{k_{0}^{2}}{2}\left(\varepsilon_{1}+\varepsilon_{2}\right)+k_{\|}^{2}$ and $B=\frac{k_{0}^{2}}{2 a}\left(\varepsilon_{1}-\varepsilon_{2}\right)$, which transforms to an Airy equation with $\eta=B^{1 / 3}(z+A / B)$ and leads to the solution

$$
\varphi^{\leqslant a}(z)=C_{1}^{\leqslant a} \mathrm{Ai}(-\eta)+C_{2}^{\leqslant a} \operatorname{Bi}(-\eta),
$$

where the superscript of the constants $C_{1,2}^{\leqslant a}$ indicates the centered layer for $|z| \leqslant a$. Analogously, one finds the constants $C_{1,2}^{>a}$ for $z>a$ and $C_{1,2}^{<-a}$ for $z<-a$. According to Maxwell's boundary conditions the scalar potential has to be continuous and differentiable, leading to the conditions

$$
\begin{aligned}
\lim _{z \rightarrow-a} \varphi^{\leqslant a}(z) & =\lim _{z \rightarrow-a} \varphi^{<-a}(z), \\
\lim _{z \rightarrow-a} \frac{d}{d z} \varphi^{\leqslant a}(z) & =\lim _{z \rightarrow-a} \frac{d}{d z} \varphi^{<-a}(z), \\
\lim _{z \rightarrow a} \varphi^{\leqslant a}(z) & =\lim _{z \rightarrow a} \varphi^{>a}(z), \\
\lim _{z \rightarrow a} \frac{d}{d z} \varphi^{\leqslant a}(z) & =\lim _{z \rightarrow a} \frac{d}{d z} \varphi^{>a}(z),
\end{aligned}
$$

resulting in a system of equations reducing the number of constants by four. By solving the system of equations, the transmission coefficient for a wave coming from the region with $\varepsilon_{1}$ to $\varepsilon_{2}$ can be extracted as

$$
\begin{aligned}
\tau_{1}= & \frac{64 \sqrt[3]{6}}{27} e^{i a\left(k_{1}+k_{2}\right)} k_{1}^{3} k_{2}^{5} a^{2} \sqrt[3]{\frac{a^{2}}{k_{0}^{16} \delta^{8} a_{1}^{4} a_{2}^{4}}}\left[I_{-1 / 3}\left(a_{2}\right) I_{-2 / 3}\left(a_{2}\right)\right. \\
& \left.-I_{2 / 3}\left(a_{2}\right) I_{1 / 3}\left(a_{2}\right)\right]\left[\left(I_{-1 / 3}\left(a_{2}\right)+I_{2 / 3}\left(a_{2}\right)\right) I_{-2 / 3}\left(a_{1}\right)\right. \\
& +\left(I_{-1 / 3}\left(a_{1}\right)-I_{2 / 3}\left(a_{1}\right)\right) I_{-2 / 3}\left(a_{2}\right)+I_{-1 / 3}\left(a_{1}\right) I_{1 / 3}\left(a_{2}\right) \\
& -I_{1 / 3}\left(a_{1}\right) I_{-1 / 3}\left(a_{2}\right)-I_{1 / 3}\left(a_{1}\right) I_{2 / 3}\left(a_{2}\right) \\
& \left.-I_{2 / 3}\left(a_{1}\right) I_{1 / 3}\left(a_{2}\right)\right]^{-1},
\end{aligned}
$$

with the modified Bessel functions $I_{v}(x)$, wave vectors in each constant region $k_{i}=\sqrt{\varepsilon_{i} k_{0}^{2}-k_{\|}^{2}}$, the arguments of the Bessel functions

$$
a_{i}=\frac{4}{3} \sqrt{-\frac{k_{i}^{6} a^{2}}{k_{0}^{4} \delta^{2}}}
$$

and the difference of the final dielectric functions $\delta=\varepsilon_{1}-$ $\varepsilon_{2}$. Due to the rotational symmetry of the profile the inverse transmission coefficient $\tilde{\tau}$ can be extracted by the exchange of the dielectric functions $\varepsilon_{1} \longleftrightarrow \varepsilon_{2}$ and changing the slope $a \mapsto-a$, which reduces to

$$
\tilde{\tau}_{1}=\frac{k_{1}^{2}}{k_{2}^{2}} \tau_{1} .
$$

This solution satisfies the transition to the Fresnel transmission by $a \mapsto 0$.

The numerical evaluation of this result showed a coincidence over a wide range of wave vectors $k_{\|}$with the Fresnel reflection coefficient. For low wave vectors $k_{\|} a<1$ the result deviates much less due to the small inhomogeneous region. For high values $k_{\|} a \gg 1$ the results are identical due to the transparency of the medium. Only in a small region, where $k_{\|} a \approx 1$, the differences are larger. By comparing the corresponding length scales of these regions one can assume that 
the error by using the Fresnel reflection coefficient instead of the exact solution is negligibly small for the considered van der Waals and Casimir interactions. By increasing the width of the inhomogeneous region its impact on the dispersion interaction increases as well, which means that the exact solution has to be taken into account.
[1] A. D. McLachlan, Proc. R. Soc. London A 271, 387 (1963).

[2] H. B. G. Casimir and D. Polder, Phys. Rev. 73, 360 (1948).

[3] P. W. Milonni, in The Quantum Vacuum, edited by P. W. Milonni (Academic Press, San Diego, 1994), pp. 217-252.

[4] H. B. G. Casimir, Indag. Math. 10, 261 (1948).

[5] R. E. Grisenti, W. Schöllkopf, J. P. Toennies, G. C. Hegerfeldt, and T. Köhler, Phys. Rev. Lett. 83, 1755 (1999).

[6] M. Arndt, O. Nairz, J. Vos-Andreae, C. Keller, G. van der Zouw, and A. Zeilinger, Nature (London) 401, 680 (1999).

[7] T. Juffmann, A. Milic, M. Müllneritsch, P. Asenbaum, A. Tsukernik, J. Tüxen, M. Mayor, O. Cheshnovsky, and M. Arndt, Nat. Nanotechnol. 7, 297 (2012).

[8] C. Brand, J. Fiedler, T. Juffmann, M. Sclafani, C. Knobloch, S. Scheel, Y. Lilach, O. Cheshnovsky, and M. Arndt, Ann. Phys. (Berlin) 527, 580 (2015).

[9] I. E. Dzyaloshinskii, E. M. Lifshitz, and L. P. Pitaevskii, Sov. Phys. Usp. 4, 153 (1961).

[10] S. Scheel and S. Buhmann, Act. Phys. Slov. 58, 675 (2008).

[11] S. Buhmann, Dispersion Forces I: Macroscopic Quantum Electrodynamics and Ground-State Casimir, Casimir-Polder and van der Waals Forces, Springer Tracts in Modern Physics (Springer, Berlin Heidelberg, 2013).

[12] F. J. P. Schuurmans, D. T. N. de Lang, G. H. Wegdam, R. Sprik, and A. Lagendijk, Phys. Rev. Lett. 80, 5077 (1998).

[13] F. J. Schuurmans, P. de Vries, and A. Lagendijk, Phys. Lett. A 264, 472 (2000).

[14] L. Onsager, J. Am. Chem. Soc. 58, 1486 (1936).

[15] J. Fiedler, P. Thiyam, A. Kurumbail, F. A. Burger, M. Walter, C. Persson, I. Brevik, D. F. Parsons, M. Boström, and S. Y. Buhmann, J. Phys. Chem. A 121, 9742 (2017).
[16] K. Autumn, Y. A. Liang, S. T. Hsieh, W. Zesch, W. P. Chan, T. W. Kenny, R. Fearing, and R. J. Full, Nature (London) 405, 681 (2000).

[17] F. A. Burger, J. Fiedler, and S. Y. Buhmann, Europhys. Lett. 121, 24004 (2018).

[18] A. Messiah, Quantum Mechanics, Dover Books on Physics (Dover Publications, Mineola, NY, 2014).

[19] S. Ribeiro, S. Y. Buhmann, T. Stielow, and S. Scheel, Europhys. Lett. 110, 51003 (2015).

[20] A. Held and M. Walter, J. Chem. Phys. 141, 174108 (2014).

[21] C. Henkel, G. Boedecker, and M. Wilkens, Appl. Phys. B 93, 217 (2008).

[22] A. Sambale, S. Y. Buhmann, D.-G. Welsch, and M.-S. Tomaš, Phys. Rev. A 75, 042109 (2007).

[23] M. S. Tomaš, Phys. Rev. A 51, 2545 (1995).

[24] W. Chew, Waves and Fields in Inhomogeneous Media (Institute of Electrical \& Electronics Engineers (IEEE), New York, 1995).

[25] P. Parashar, K. A. Milton, Y. Li, H. Day, X. Guo, S. A. Fulling, and I. Cavero-Peláez, Phys. Rev. D 97, 125009 (2018).

[26] I. Griniasty and U. Leonhardt, Phys. Rev. A 96, 032123 (2017).

[27] K. A. Milton, Phys. Rev. D 84, 065028 (2011).

[28] K. A. Milton, J. Opt. Soc. Am. B 36, C41 (2019).

[29] A. Derevianko, S. G. Porsev, and J. F. Babb, At. Data Nucl. Data Tables 96, 323 (2010).

[30] M. Elbaum and M. Schick, Phys. Rev. Lett. 66, 1713 (1991).

[31] J. Vogt and S. Alvarez, Inorg. Chem. 53, 9260 (2014).

[32] Y. Zhang and Z. Xu, Am. Mineral. 80, 670 (1995).

[33] U. Hohm and A. J. Thakkar, J. Phys. Chem. A 116, 697 (2012).

[34] J. Jackson, Classical Electrodynamics (Wiley, New York, 1998). 